\title{
THE AFOE-A NEW INSTRUMENT FOR ASTEROSEISMOLOGY
}

\author{
R. W. NOYES
}

Harvard-Smithsonian Center for Astrophysics, Cambridge, MA, USA

\author{
T. M. BROWN
}

High Altitude Observatory, Boulder, CO, USA

S. HORNER

University of Chicago, Chicago, IL, USA

\section{S. KORZENNIK, P. NISENSON}

Harvard-Smithsonian Center for Astrophysics, Cambridge, MA, USA

ABSTRACT We describe technical aspects and performance characteristics of an Advanced Fiber Optic Spectrograph (AFOE), planned for the Mt. Hopkins $1.5 \mathrm{~m}$ telescope for asteroseismology of sun-like stars.

Determining the oscillation spectra of sun-like stars by Doppler techniques requires measuring velocity fluctuations to better than $1 \mathrm{~m} \mathrm{~s}^{-1}$ within about one night's observation. Straightforward considerations (e.g. Brown 1990) imply that the photon noise-limited precision attainable by measuring a single spectrum line is approximately $\delta v_{r m s} \simeq c w /\left[\lambda d\left(N_{p i x} I_{c}\right)^{1 / 2}\right]$, where $\delta v_{r m s}$ is the rms Doppler noise, $c, w, d$, and $\lambda$ are the speed of light, the apparent linewidth (including instrumental broadening), the relative line depth, and the line wavelength, $N_{p i x}$ is the number of detector pixels spanning the line, and $I_{c}$ is the continuum intensity measured in photoelectrons per pixel. For parameter values that are typically found in bright-star spectroscopy $(\lambda=500 \mathrm{~nm}, w=0.01 \mathrm{~nm}$, $\left.d=0.6, N_{p i x}=3, I_{c}=10^{5}\right)$, one obtains $\delta v_{r m s}=11 \mathrm{~m} \mathrm{~s}^{-1}$. For bright stars and moderate-sized telescopes, an exposure $I_{c}$ can be reached in a few minutes.

This level of precision is still inadequate for seismology of sun-like stars. The Advanced Fiber Optic Echelle (AFOE) spectrograph achieves a substantial gain in precision through several approaches: (1) We increase $N_{\text {pix }}$ by increasing the number of lines measured. This is achieved with a cross-dispersed echelle, coupled with a large-format $\left(2048^{2} 15-\mu\right.$ pixel $)$ CCD. (2) We minimize the apparent linewidth $w$ by maximizing the spectral resolution. Spectral resolution must be traded off against spectral coverage; this is optimized for maximum S/N when observing slowly-rotating Sun-like stars. This results in coverage in one exposure of about $55 \%$ of the wavelength range between 470 and $610 \mathrm{~nm}$, with 
FIBER SCRAMBLER / SLICER

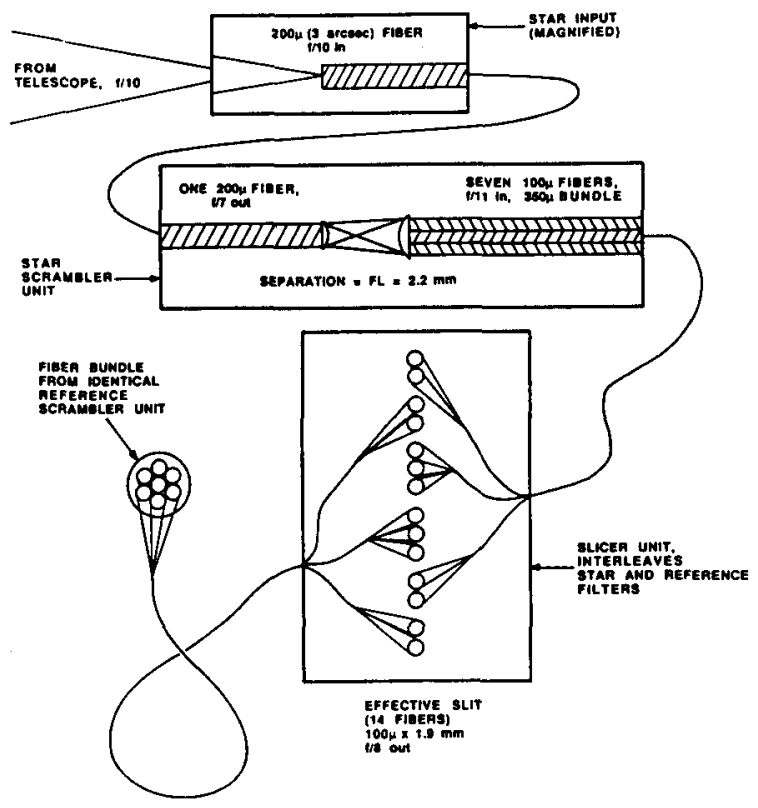

Figure 1: The Fiber Scrambler/Slicer receives the $f / 10$ star image in a $200-\mu$ (3 arcsec) fiber [top] and relays it to the star-scrambler unit [center], where complete scrambling is effected by interchanging positional and angular coordinates of the star image (see text), and where the light is re-imaged at $f / 11$ into a bundle of seven $100-\mu$ fibers. An identical arrangement is provided for the reference image. The total of $14100-\mu$ fibers are then brought to the AFOE and interleaved [bottom] along the entrance slit, where they produce an $\mathrm{f} / 8$ effective slit image. The two input fibers may be switched between star and reference to difference out any residual dissimilarities between the two optical paths.

a resolution of 58000 . (3) We maximize $I_{c}$ for a given exposure time by use of a fiber-optic image slicer to avoid slit losses, even in indifferent seeing; the image slicer(see Figure 1) maps the $200 \mu(3 \mathrm{arcsec})$ telescope entrance aperture onto a $100 \mu$ wide spectrograph entrance slit. We also use high-reflectance optical coatings wherever possible. The peak efficiency of the system (including telescope transmission, fiber coupling, spectrograph efficiency, and detector QE) is about $1.5 \%$. This system on a $1.5 \mathrm{~m}$ telescope should allow Doppler measurements with photon-limited precision of $80 \mathrm{~cm} \mathrm{~s}^{-1}$ in a 60 s exposure on a V=0 G-type star (i.e. $50 \mathrm{~cm} \mathrm{~s}^{-1}$ in 5 hours total integration on a similar $\mathrm{V}=5 \mathrm{star}$ ). We have already obtained near photon-limited precision with the Penn State Fiber Optic Echelle (FOE) on the KPNO 2.1m telescope (Brown et al. 1991, Brown 1990) so we are confident that the expected level of precision is realistic.

A $1 \mathrm{~m} \mathrm{~s}^{-1}$ Doppler shift represents a motion of the spectrum on the detector of only $3 \times 10^{-4}$ of a 15 -micron pixel. Not only must the precision of individual Doppler measurements permit detecting such shifts, but measurement stability must be maintained to this level over times significantly longer than the period of the oscillation modes being measured. Our approach is to provide as great mechanical stability as practical in the AFOE, and to use continuous wavelength calibration to correct for residual instrumental drifts. 
To maximize mechanical stability, the spectrograph will be mounted on a vibration-isolated optical table, connected to the telescope by an optical fiber, and enclosed in a thermally-controlled housing within a dedicated room. The CCD detector will be continuously cooled thermoelectrically, to avoid transient behavior accompanying filling with cryogens.

To calibrate out residual instrumental drifts, the AFOE incorporates two distinct continuous calibration methods: (1) An iodine vapor absorption cell may be inserted into the beam of starlight, imposing a spectrum of thousands of molecular lines which act as a well-defined wavelength reference (e.g. Marcy and Butler 1992); (2) A separate optical fiber will bring light from a reference emission-line source into the spectrograph adjacent to the stellar input fiber. To minimize effects of different optical paths through the spectrograph, the fiber image slicer (Figure 1) will interleave the stellar and reference light within each echelle order. Any remaining differences may be calibrated out by periodically interchanging the fibers carrying stellar and reference light.

One important potential source of noise in Doppler measurements with the AFOE is variable illumination of the collimator due to imperfect seeing or guiding. This problem is not completely eliminated by the scrambling action of a single optical fiber, since high-quality fibers do not alter the off-axis angle of light rays that pass through them. To solve this problem, the AFOE incorporates a double fiber scrambler (Brown, 1990), in which a pair of fibers is coupled together using a pair of microlenses separated by their common focal length; the off-axis angle in the first fiber is then mapped into radial position on the face of the second, and subsequently scrambled within the second fiber. The double scrambler will be incorporated into the fiber image slicer (see Fig. 1), to minimize light losses.

The Sun is a useful object for calibrating the AFOE precision and stability, since it is very bright and has a very well-known radial velocity relative to the Earth. To allow regular observations of the Sun, a heliostat and optical fiber feed will bring unimaged sunlight into the spectrograph in the same configuration and brightness as a $V \approx-5$ star. Solar observations will: provide an independent stellar wavelength standard; allow characterization of pure instrumental drifts (since the high signal level will virtually eliminate photon noise); and through detection of solar p-modes provide a known calibration source for stellar oscillation mode amplitudes.

\section{REFERENCES}

Brown, T.M. 1990, in CCD's in Astronomy, A.S.P. Conference Series 8, p. 335. Brown,T.M., Gilliland, R.L., Noyes, R.W., and Ramsey, L.W. 1991, Ap. J. 368, 599.

Marcy, G.W., and Butler, R.P. 1992, P.A.S.P. 104, 270. 